Journal of Mathematics and Statistics Studies

ISSN: 2709-4200

DOI: $10.32996 / j m s s$

Journal Homepage: www.al-kindipublisher.com/index.php/jmss

\title{
Fundamental Results on Determining Matrices for a Certain Class of Hereditary Systems
}

\author{
Onyekachukwu Henry lkeh ${ }^{\mathbf{9}}$ (D) $\triangle$ and Chukwunenye Ukwu' 9 (D), \\ ${ }^{7}$ Department of Basic Sciences, Federal School of Medical Laboratory Technology (Science) Jos, Nigeria \\ ${ }^{2}$ Department of Mathematics, University of Jos, Nigeria \\ $\triangle$ Corresponding Author: Onyekachukwu Henry Ikeh, E-mail: ikehhenry245@gmail.com
}

\section{ARTICLE INFORMATION ABSTRACT}

Received: April 08, 2021

Accepted: June 01, 2021

Volume: 2

Issue: 1

DOI: $10.32996 / j m s s .2021 .2 .1 .7$

\section{KEYWORDS}

Determining, Controllability, Euclidean, Generic, double time-

delay, matrices, control systems
Three major tools are required to investigate the controllability of control systems, namely, determining matrices, index of control systems and controllability Grammian. Determining matrices are the preferred choice for autonomous control systems due to the fact that they are devoid of integral operators in their computations. This article developed the structure of certain parameter-ordered determining matrices of generic double time-delay linear autonomous functional differential control systems, with a view to obtaining the controllability matrix associated with the rank condition for Euclidean controllability of the system. Expressions for the relevant determining matrices were formulated and it was established that the determining matrices for double time-delay linear autonomous functional differential control systems do not exist if one of the time-delays is not an integer multiple of the other paving the way for the investigation of the Euclidean controllability of generic double time-delay control systems.

\section{Introduction}

Controllability results for specific types of hereditary systems with diversity in treatment approaches are quite prevalent in control literature. Angell (1980) discussed controllability of nonlinear hereditary systems, using a fixed point approach, (Balachandran, 1986) discussed controllability of nonlinear systems with delays in both state and control variables using a constructive control approach and an appeal to Arzela-Ascoli, and Shauder fixed point theorems to guarantee the existence and admissibility of such controls. Balachandran (1992) and Balachandran and Balasubramaniam (1993) studied the controllability of Volterra Integro-differential systems. Banks and Kent (1972) discussed controllability of functional differential equations of retarded and neutral types to targets in function space; Dauer and Gahl (1977) looked at controllability of nonlinear delay systems; Onwuatu (1984) studied null controllability in function space of nonlinear neutral differential systems with limited controls; Underwood R. G. and Chukwu (1988) investigated null controllability of nonlinear neutral differential equations, to mention just a few.

Chukwu (2001) further extended this research field by formulating differential models and neutral systems for controlling the wealth of nations. His work derives from the economic principles of the dynamics of national income, interest rate, employment, the value of the capital stock prices and cumulative balance of payments. Chukwu used a Volterra neutral integro-differential game of pursuit where the quarry control is government intervention in the form of taxation, control of money and supply tariffs. Other notable results focus on integro-differential equations and impulsive differential equations with finite and infinite delays (Chang \& Chalishajar, 2008; Selvi \& Mallika, 2012). These works and others appropriate relevant existence and uniqueness of solution theorems which this study relies on.

Gabasov and Kirillova (1976) first introduced the concept of determining matrices by formulating necessary and sufficient conditions for the Euclidean controllability of the single time-delay system: $\dot{x}(t)=A x(t)+B x(t-h)+C u(t)$ with



Your gateway to world-class research

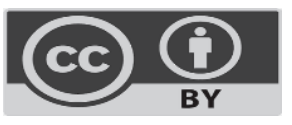

Published by Al-Kindi Center for Research and Development. Copyright (c) the author(s). This open access article is distributed under a Creative Commons Attribution (CC-BY) 4.0 license 
piecewise continuous controls using a sequence of matrices $Q_{k}(s), k=0,1, \ldots s$ real. But the investigation of the dependence of $Q_{\infty}\left(t_{1}\right)$ on $Q_{0}(s), Q_{1}(s), \ldots, Q_{n-1}(s)$ which is very crucial to establishing Euclidean controllability was not fully addressed.

Ukwu (1992) developed computational criteria for the Euclidean controllability of the above single time-delay system using the determining matrices with a very simple structure, effectively eliminating the afore-mentioned drawback. However, a major drawback of his major result is that it relied on (Manitius, 1978) for the necessary and sufficient conditions for the Euclidean controllability of the delay system, stated in terms of the transition matrices, which until (Ukwu, 1992) was a herculean or almost impossible task to obtain. Ukwu (2014) states that multiple summations and permutation of objects were used to derive the expressions for the determining matrices. By using the change of variables technique, deft application of mathematical induction principles and careful avoidance of some induction pitfalls, he was able to obtain the structure of the determining matrices for the single-delay neutral control model: $\frac{d}{d t}\left[x(t)-A_{-1} x(t-h)\right]=A_{0} x(t)+A_{1} x(t-h) B u(t), t \geq 0$, without which the computational investigation of Euclidean controllability would be impossible.

Ukwu (2016a) pioneered the introduction of the least integer function in the statement and proof of the necessary and sufficient conditions for the Euclidean controllability of linear hereditary systems of double time-delay: $\dot{x}(t)=A_{0} x(t)+A_{1} x(t-h)+A_{2} x(t-2 h)+B u(t) ; t \geq 0$ with an initial function: $x(t)=\phi(t), t[-2 h, 0], h>0$; this made the controllability matrix in (Gabasov \& Kirillova, 1976) quite computable and eliminates any ambiguity that could arise in its application, he also incorporated the characterization of Euclidean controllability in terms of the indices of control systems and appropriated Taylor's theorem as an indispensable tool. The article provided an illustrative example on the computation of the controllability matrices and stated the implication of the result for Euclidean controllability.

However, these works were all limited to single time-delay control systems and double time-delay control systems of nongeneric delays in the state variable. This study will extend the research activity to a class of generic double time-delay control systems when the delay in the state variable is non-commensurate or generic with certain conditions imposed on the delay values to avoid ambiguity. The expected results will add to the already existing body of knowledge in this field and will have practical implications.

\section{Methodology}

Consider the generic double time-delay linear autonomous control system:

$$
\begin{aligned}
& \dot{x}(t)=A_{0} x(t)+A_{1} x\left(t-h_{1}\right)+A_{2} x\left(t-h_{2}\right)+B u(t) ; t \geq 0 \\
& x(t)=\phi(t), t \in[-h, 0], h_{1}<h_{2}, h_{1} \neq h_{2}, h=\max \left\{h_{1}, h_{2}\right\}>0
\end{aligned}
$$

where $A_{0}, A_{1}, A_{2}$ are $n \times n$ constant matrices with real entries, $B$ is an $n \times m$ constant matrix with real entries. The initial function $\phi$ is in $C\left([-h, 0], \mathbf{R}^{n}\right)$, the space of continuous functions from $[-h, 0]$ into the real $\mathrm{n}$-dimensional Euclidean space, $\mathbf{R}^{n}$ with norm defined by $\|\phi\|=\sup _{t \in[-h, 0]}|\phi(t)|$ (the sup norm). The control $u$ is in the space $L_{\infty}\left(\left[0, t_{1}\right], \mathbf{R}^{n}\right)$, the space of essentially bounded measurable functions taking $\left[0, t_{1}\right]$ into $\mathbf{R}^{n}$ with norm $\|\phi\|=e s s \sup _{t \in[-h, 0]}|\phi(t)|$. Any control $u \in L_{\infty}\left(\left[0, t_{1}\right], \mathbf{R}^{n}\right)$ will be referred to as an admissible control.

2.1 Preliminaries on the Partial Derivatives $\frac{\partial^{k} X(\tau, t)}{\partial \tau^{k}}, k=0,1, \ldots$

Let $t, \tau \in\left[0, t_{1}\right]$. For fixed t, let $\tau \rightarrow X(\tau, t)$ satisfy the matrix differential equation:

$$
\frac{\partial}{\partial \tau} X(\tau, t)=-X(\tau, t) A_{0}-X\left(\tau+h_{1}, t\right) A_{1}-X\left(\tau+h_{2}, t\right) A_{2}
$$


for $0<\tau<t, \tau \neq t-k h, h=\max \left\{h_{1}, h_{2}\right\}, k=0,1, \ldots$ where $X(\tau-t)=\left\{\begin{array}{l}I_{n}, \tau=t \\ 0, \tau>t\end{array}\right.$

Chukwu in [7] has the properties of $X(t, \tau)$ clearly stated; of particular importance is the fact that $\tau \rightarrow X(\tau, t)$ is analytic on the intervals $\left(t_{1}-(j+1) h, t_{1}-j h\right), j=0,1, \ldots, t_{1}-(j+1) h>0$. Any such $\tau \in\left(t_{1}-(j+1) h, t_{1}-j h\right)$ is called a regular point of $\tau \rightarrow X(\tau, t)$.

Let $X^{k}(\tau, t)$ denote $\frac{\partial^{k}}{\partial \tau^{k}} X\left(\tau, t_{1}\right)$, the $k^{\text {th }}$ partial derivative of $X\left(\tau, t_{1}\right)$ with respect to $\tau$ where $\tau \in\left(t_{1}-(j+1) h, t_{1}-j h\right) ; \quad j=0,1, \ldots, r \quad$ for $\quad$ some integer $\quad r \quad$ such that $t_{1}-(r+1) h>0$. Write $X^{(k+1)}\left(\tau, t_{1}\right)=\frac{\partial}{\partial \tau} X^{k}\left(\tau, t_{1}\right) \quad$ and define $\Delta X^{k}\left(t_{1}-j h, t_{1}\right)=X^{k}\left(\left(t_{1}-j h\right)^{-}, t_{1}\right)-X^{k}\left(\left(t_{1}-j h\right)^{+}, t_{1}\right) \quad$ for $k=0,1, \ldots ; j=0,1, \ldots ; t_{1}-j h>0$ where $X^{k}\left(\left(t_{1}-j h\right)^{-}, t_{1}\right)$ and $X^{k}\left(\left(t_{1}-j h\right)^{+}, t_{1}\right)$ denote respectively the left and right hand limits of $X\left(\tau, t_{1}\right)$ at $\tau=t_{1}-j h$.

\subsection{Investigation of the Expressions and Structure of the Determining Matrices for (1)}

Let $Q_{k}(s)$ be an $n \times n$ matrix function defined by:

$$
Q_{k}(s)=A_{0} Q_{k-1}(s)+A_{1} Q_{k-1}\left(s-h_{1}\right)+A_{2} Q_{k-1}\left(s-h_{2}\right)
$$

for $k=1,2, \ldots ; s>0$, with initial conditions:

$$
\begin{aligned}
& Q_{0}(0)=I_{n} \\
& Q_{0}(s)=0 ; s \neq 0
\end{aligned}
$$

These initial conditions guarantee the unique solvability of (4) (Gabasov \& Kirillova, 1976).

\subsubsection{Preliminary Lemma on Determining Matrices for (1) $Q_{k}(s), s \in \mathbf{R}$}

i. $Q_{k}(0)=A_{0}^{k}$

ii. $Q_{k}(s)=0$ if $s<0$

iii. $Q_{k}(s)=0$ if $s \neq r h_{1}, s \neq r h_{2}, s \neq r h_{1}+q h_{2}$ for any integer $r, q \neq 0$

iv. $\quad Q_{k}\left(h_{1}\right)=\sum_{\left(v_{1}, \ldots, v_{k}\right) \in P_{0(k-1),(1), 2(0)}} \prod_{i=1}^{k} A_{v_{i}} ; k \geq 1$

v. $Q_{k}\left(h_{1}-r h_{2}\right)=0$ for all $k \geq 1, r \neq 0$ integer

vi. $Q_{1}\left(j h_{1}\right)=\left\{\begin{array}{l}A_{0} \text { if } j=0 \\ A_{1} \text { if } j=1 \\ A_{2} \text { if } h_{2}=j h_{1}, j=\frac{h_{2}}{h_{1}}=; j \geq 2 \\ 0, \text { otherwise }\end{array}\right.$

vii. $\quad Q_{k}\left(j h_{1}\right)=0$ if $h_{2} \neq r h_{1}$ for any integer $j, r>1$

viii. $\quad X^{(k)}\left(t_{1}^{-}, t_{1}\right)=\left(-A_{0}\right)^{k}=(-1)^{k} A_{0}^{k}, k=1,2,3, \ldots$ 
ix. $\quad X^{(k)}\left(t_{1}^{+}, t_{1}\right)=0$

x. $\Delta X\left(t_{1}-j h, t_{1}\right)=\left\{\begin{array}{l}I_{n} \text { if } j=0 \\ 0, \text { otherwise }\end{array}\right.$

Proof:

i. $\quad Q_{k}(s)=A_{0} Q_{k-1}(s)+A_{1} Q_{k-1}\left(s-h_{1}\right)+A_{2} Q_{k-1}\left(s-h_{2}\right)$

For $k=1$ :

$Q_{1}(0)=A_{0} Q_{0}(0)+A_{1} Q_{0}\left(-h_{1}\right)+A_{2} Q_{0}\left(-h_{2}\right)=A_{0}$

(Since $Q_{0}(0)=I_{n}, Q_{0}\left(-h_{1}\right)=0$, if $-h_{1} \neq 0$ and $Q_{0}\left(-h_{2}\right)=0$, if $-h_{2}=0$ )

So, (i) is true for $\mathrm{k}=1$; we next assume (i) is true for $1<k \leq n$ for any integer $\mathrm{n}$ such that $Q_{n}(0)=A_{0}^{n}$, then

$$
\begin{aligned}
Q_{n+1}(0)=A_{0} Q_{n}(0)+A_{1} Q_{n}\left(-h_{1}\right) & +A_{2} Q_{n}\left(-h_{2}\right) \\
= & A_{0} A_{0}^{n}+A_{1} Q_{n}\left(-h_{1}\right)+A_{2} Q_{n}\left(-h_{2}\right)
\end{aligned}
$$

Assertion 1: $Q_{k}(s)=0$ if $s<0$

Proof: For $k=1$

$$
Q_{1}(s)=A_{0} Q_{0}(s)+A_{1} Q_{0}\left(s-h_{1}\right)+A_{2} Q_{0}\left(s-h_{2}\right)=0
$$

(Since $s \neq 0$ and $h_{1}, h_{2}>0$, it implies that $s \neq h_{1}$ and $s \neq h_{2}$; and from the initial condition)

So the assertion is true for $k=1$, we assume it is true for $1<k \leq n$ for some integer $\mathrm{n}$ such that $Q_{n}(s)=0$, then

$$
Q_{n+1}(s)=A_{0} Q_{n}(s)+A_{1} Q_{n}\left(s-h_{1}\right)+A_{2} Q_{n}\left(s-h_{2}\right)=0
$$

(Since $Q_{n}(s)=0$ and $s \neq h_{1}, h_{2}$ implies $s-h_{1}, s-h_{2}<0$ thus $Q_{n}\left(s-h_{1}\right), Q_{n}\left(s-h_{2}\right)=0$ )

Hence by the induction hypothesis: $Q_{k}(s)=0$ if $s<0$ which proves the assertion; we proceed to conclude the proof of (i):

$$
Q_{n+1}(0)=A_{0} A_{0}^{n}+A_{1} Q_{n}\left(-h_{1}\right)+A_{2} Q_{n}\left(-h_{2}\right)=A_{0}^{n+1} \text { (Since } Q_{k}(s)=0 \text { if } s<0 \text { ) }
$$

Hence by the induction hypothesis: $Q_{k}(0)=A_{0}^{k}$

ii. $Q_{k}(s)=0$ if $s<0$ (The proof is embedded in (i) above)

iii. Let $k=1$ and $s \neq r h_{1}$ or $s \neq q h_{2}, r, q$ integers, then

$$
Q_{1}(s)=A_{0} Q_{0}(s)+A_{1} Q_{0}\left(s-h_{1}\right)+A_{2} Q_{0}\left(s-h_{2}\right)=0
$$

(Since $s \neq 0$ and $h_{1}, h_{2}>0$, it implies that $s \neq h_{1}$ and $s \neq h_{2}$; and from the initial condition)

So the assertion is true for $k=1$, we assume it is true for $1<k \leq n$ for some integer $\mathrm{n}$ such that $Q_{n}(s)=0$ if $s \neq r h_{1}, s \neq q h_{2}, s \neq r h_{1}+q h_{2}$ then

$$
Q_{n+1}(s)=A_{0} Q_{n}(s)+A_{1} Q_{n}\left(s-h_{1}\right)+A_{2} Q_{n}\left(s-h_{2}\right)
$$

Assertion 2: $Q_{k}\left(s-h_{1}\right)=0$ if $s \neq r h_{1}, s \neq r h_{1}+q h_{2}, r, q$ integers

Proof: Let $k=1$, then

$$
Q_{1}\left(s-h_{1}\right)=A_{0} Q_{0}\left(s-h_{1}\right)+A_{1} Q_{0}\left(s-2 h_{1}\right)+A_{2} Q_{0}\left(s-h_{1}-h_{2}\right)=0
$$

(Since $s \neq h_{1}, s \neq 2 h_{1}, s \neq h_{1}+h_{2}$ implies $Q_{0}\left(s-h_{1}\right)=Q_{0}\left(s-2 h_{1}\right)=Q_{0}\left(s-h_{1}-h_{2}\right)=0$ ) 
So the assertion is true for $k=1$, we assume it is true for $1<k \leq n$ for some integer $\mathrm{n}$ such that $Q_{n}\left(s-h_{1}\right)=0$ if $s \neq r h_{1}, s \neq r h_{1}+q h_{2}, r, q$ integers, then

$$
Q_{n+1}\left(s-h_{1}\right)=A_{0} Q_{n}\left(s-h_{1}\right)+A_{1} Q_{n}\left(s-2 h_{1}\right)+A_{2} Q_{n}\left(s-h_{1}-h_{2}\right)=0
$$

(Since $s \neq h_{1}, s \neq 2 h_{1}, s \neq h_{1}+h_{2}$ implies $Q_{n}\left(s-h_{1}\right)=Q_{n}\left(s-2 h_{1}\right)=Q_{n}\left(s-h_{1}-h_{2}\right)=0$ )

Hence by the induction hypothesis: $Q_{k}\left(s-h_{1}\right)=0$ if $s \neq r h_{1}, s \neq r h_{1}+q h_{2}, r, q$ integers which proves the assertion. Similarly,

Assertion 3: $Q_{k}\left(s-h_{2}\right)=0$ if $s \neq q h_{2}, s \neq r h_{1}+q h_{2}, r, q$ integers

Proof: Let $k=1$ then

$$
Q_{1}\left(s-h_{2}\right)=A_{0} Q_{0}\left(s-h_{2}\right)+A_{1} Q_{0}\left(s-h_{2}-h_{1}\right)+A_{2} Q_{0}\left(s-2 h_{2}\right)=0
$$

(Since $s \neq h_{2}, s \neq 2 h_{2}, s \neq h_{1}+h_{2}$ implies $Q_{0}\left(s-h_{2}\right)=Q_{0}\left(s-2 h_{2}\right)=Q_{0}\left(s-h_{1}-h_{2}\right)=0$ )

So the assertion is true for $k=1$, we assume it is true for $1<k \leq n$ for some integer $\mathrm{n}$ such that $Q_{n}\left(s-h_{2}\right)=0$ if $s \neq q h_{2}, s \neq r h_{1}+q h_{2}, r, q$ integers, then

$$
Q_{n+1}\left(s-h_{2}\right)=A_{0} Q_{n}\left(s-h_{2}\right)+A_{1} Q_{n}\left(s-h_{2}-h_{1}\right)+A_{2} Q_{n}\left(s-2 h_{2}\right)=0
$$

(Since $s \neq h_{2}, s \neq 2 h_{2}, s \neq h_{1}+h_{2}$ implies $Q_{n}\left(s-h_{2}\right)=Q_{n}\left(s-2 h_{2}\right)=Q_{n}\left(s-h_{1}-h_{2}\right)=0$ )

Hence by induction hypothesis: $Q_{k}\left(s-h_{2}\right)=0$ if $s \neq q h_{2}, s \neq r h_{1}+q h_{2}, r, q$ integers which proves the assertion.

Combining the Assertions 2 and 3 will yield:

$$
Q_{n+1}(s)=A_{0} Q_{n}(s)+A_{1} Q_{n}\left(s-h_{1}\right)+A_{2} Q_{n}\left(s-h_{2}\right)=0
$$

And thus by the induction hypothesis:

$Q_{k}(s)=0$ if $s \neq r h_{1}, s \neq r h_{2}, s \neq r h_{1}+q h_{2}$ for any integer $r, q \neq 0$, which proves (iii).

iv. Let $k=1$, then

$$
Q_{1}\left(h_{1}\right)=A_{0} Q_{0}\left(h_{1}\right)+A_{1} Q_{0}(0)+A_{2} Q_{0}\left(h_{1}-h_{2}\right)=A_{1}=\sum_{v_{1} \in P_{0(0),(1), 2(0)}} A_{v_{1}}
$$

(Since $h_{1} \neq 0, h_{2} \neq 0$ implies $Q_{0}\left(h_{1}\right)=Q_{0}\left(h_{1}-h_{2}\right)=0$ and $Q_{0}(0)=I_{n}$ )

So the assertion is true for $k=1$, we assume it is true for $1<k \leq n$ for some integer $\mathrm{n}$ such that

$$
\begin{aligned}
& Q_{n}\left(h_{1}\right)=\sum_{\left(v_{1}, \ldots, v_{n}\right) \in P_{0(n-1),(1), 2(0)}} \prod_{i=1}^{n} A_{v_{i}} \text {, then } \\
& Q_{n+1}\left(h_{1}\right)=A_{0} Q_{n}\left(h_{1}\right)+A_{1} Q_{n}(0)+A_{2} Q_{n}\left(h_{1}-h_{2}\right) \\
& =A_{0} \sum_{\left(v_{1}, \ldots, v_{n}\right) \in P_{0(n-1),(1), 2(0)}} \prod_{i=1}^{n} A_{v_{i}}+A_{1} A_{0}^{n}+A_{2} Q_{n}\left(h_{1}-h_{2}\right)
\end{aligned}
$$

Assertion 4: $Q_{k}\left(h_{1}-r h_{2}\right)=0$ for all $k \geq 1, r \neq 0$ integer

Proof: Let $k=1$ then

$$
Q_{1}\left(h_{1}-r h_{2}\right)=A_{0} Q_{0}\left(h_{1}-r h_{2}\right)+A_{1} Q_{0}\left(-r h_{2}\right)+A_{2} Q_{0}\left(h_{1}-(r+1) h_{2}\right)=0
$$

(Since $h_{2}>h_{1}$ so $h_{1} \neq r h_{2}$ and $h_{1} \neq(r+1) h_{2}$, thus $Q_{0}\left(h_{1}-r h_{2}\right)=Q_{0}\left(h_{1}-(r+1) h_{2}\right)=0=Q_{0}\left(-r h_{2}\right)$ So the assertion is true for $k=1$, we assume it is true for $1<k \leq n$ for some integer $\mathrm{n}$ such that $Q_{n}\left(h_{1}-r h_{2}\right)=0, r$ integer, then

$$
Q_{n+1}\left(h_{1}-r h_{2}\right)=A_{0} Q_{n}\left(h_{1}-r h_{2}\right)+A_{1} Q_{n}\left(-r h_{2}\right)+A_{2} Q_{n}\left(h_{1}-(r+1) h_{2}\right)=0
$$


(Since $Q_{n}\left(h_{1}-r h_{2}\right)=Q_{n}\left(h_{1}-(r+1) h_{2}\right)=0$ and $Q_{n}\left(-r h_{2}\right)=0$ )

Hence by the induction hypothesis: $Q_{k}\left(h_{1}-r h_{2}\right)=0$ for all $k \geq 1, r \neq 0$ integer which proves the assertion.

So to conclude the proof, we have:

$$
\begin{aligned}
& Q_{n+1}\left(h_{1}\right)=A_{0} \sum_{\left(v_{1}, \ldots, v_{n}\right) \in P_{O(n-1),(1), 2(0)}} \prod_{i=1}^{n} A_{v_{i}}+A_{1} A_{0}^{n}+A_{2} .0 \\
& \left.=\sum_{\left(v_{1}, \ldots, v_{n+1}\right) \in P_{O(n),(1), 2(0)}} \prod_{i=1}^{n+1} A_{v_{i}} \text { (with a leading } A_{0}\right)+\sum_{\left(v_{1}, \ldots, v_{n+1}\right) \in P_{O(n),(1), 2(0)}} \prod_{i=1}^{n+1} A_{v_{i}} \text { (with a leading } A_{1} \text { ) }
\end{aligned}
$$

Thus: $Q_{n+1}\left(h_{1}\right)=\sum_{\left(v_{1}, \ldots, v_{n+1}\right) \in P_{((n), 1(1), 2(0)}} \prod_{i=1}^{n+1} A_{v_{i}}$

Hence by the induction hypothesis: $Q_{k}\left(h_{1}\right)=\sum_{\left(v_{1}, \ldots, v_{k}\right) \in P_{(k-1),(1), 2(0)}} \prod_{i=1}^{k} A_{v_{i}} ; k \geq 1$ which proves (iv).

v. (The proof is embedded in (iv) above)

vi. $Q_{1}(0)=A_{0}$ and $Q_{1}\left(h_{1}\right)=A_{1}$ by Lemma (i) and (vi) respectively; then for $j \geq 2$

$$
Q_{1}\left(j h_{1}\right)=A_{0} Q_{0}\left(j h_{1}\right)+A_{1} Q_{0}\left([j-1] h_{1}\right)+A_{2} Q_{0}\left(j h_{1}-h_{2}\right)=A_{2} Q_{0}\left(j h_{1}-h_{2}\right)
$$

(Since $j, j-1>0$ then $Q_{0}\left(j h_{1}\right)=Q_{0}\left([j-1] h_{1}\right)=0$ from the initial condition ) and

$$
Q_{0}\left(j h_{1}-h_{2}\right)=\left\{\begin{array}{l}
I_{n} \text { if } h_{2}=j h_{1} \Rightarrow j=\frac{h_{2}}{h_{1}} \text { 星 } \\
0 \text { otherwise }
\end{array}\right.
$$

Hence, $Q_{1}\left(j h_{1}\right)=\left\{\begin{array}{l}A_{2} \text { if } h_{2}=j h_{1} \Rightarrow j=\frac{h_{2}}{h_{1}} \text {; } ; \text { which proves the lemma. } \\ 0 \text { otherwise }\end{array}\right.$

vii. Let $k=1$ and let $h_{2} \neq r h_{1}$ for any integer $r>1$. Then

$$
Q_{1}\left(j h_{1}\right)=A_{0} Q_{0}\left(j h_{1}\right)+A_{1} Q_{0}\left([j-1] h_{1}\right)+A_{2} Q_{0}\left(j h_{1}-h_{2}\right)
$$

From the initial condition; since $h_{2} \neq r h_{1}$ and $j, r>1$, then $Q_{0}\left(j h_{1}\right)=0$ if $j h_{1} \neq 0, Q_{0}\left([j-1] h_{1}\right)=0$ if $[j-1] h_{1} \neq 0$, and $Q_{0}\left(j h_{1}-h_{2}\right)=0$ if $h_{2} \neq j h_{1}$; thus

$$
Q_{1}\left(j h_{1}\right)=0 \text { for all } j, r>1, h_{2} \neq r h_{1}
$$

Assume that this is true for $2 \leq k \leq n$ for some integer $n$. Then

$$
Q_{n+1}\left(j h_{1}\right)=A_{0} Q_{n}\left(j h_{1}\right)+A_{1} Q_{n}\left([j-1] h_{1}\right)+A_{2} Q_{n}\left(j h_{1}-h_{2}\right)=0
$$

by the induction hypothesis. Hence $Q_{k}\left(j h_{1}\right)=0$ if $h_{2} \neq r h_{1}$ for any integer $j_{1} r>1$. This result establishes that the determining matrices become zero if the larger delay term $h_{2}$ is not an integer multiple of the smaller delay term $h_{1}$.

viii.

$$
X^{(k)}\left(t_{1}^{-}, t_{1}\right)=\left.\frac{\partial}{\partial \tau} X^{(k-1)}\left(\tau, t_{1}\right)\right|_{\tau=t_{1}^{-}}
$$

For $k=1$, this yields

$$
\begin{aligned}
X^{(1)}\left(t_{1}^{-}, t_{1}\right)=- & X\left(t_{1}^{-}, t_{1}\right) A_{0}-X\left(\left(t_{1}+h_{1}\right)^{-}, t_{1}\right) A_{1}-X\left(\left(t_{1}+h_{2}\right)^{-}, t_{1}\right) A_{2} \\
& \left.=-A_{0}-0 . A_{1}-0 . A_{2}=-A_{0} \text { (Since } t_{1}+h_{1}>t_{1} \text { and } t_{1}+h_{2}>t_{1}\right)
\end{aligned}
$$


Therefore $X^{(1)}\left(t_{1}^{-}, t_{1}\right)=-A_{0}=(-1) A_{0}$. Thus (ix) is true for $k=1$ Note that for $\tau$ sufficiently close to $t_{1}, \tau+h_{1}>t_{1}$ and $\tau+h_{2}>t_{1}$ For $k=2$ :

$$
\begin{aligned}
X^{(2)}\left(t_{1}^{-}, t_{1}\right)=- & {\left.\left[\frac{\partial}{\partial \tau} X^{(1)}\left(\tau, t_{1}\right)\right]\right|_{\tau=t_{1}^{-}}=X^{(1)}\left(t_{1}^{-}, t_{1}\right) A_{0}-X^{(1)}\left(\left(t_{1}+h_{1}\right)^{-}, t_{1}\right) A_{1}-X^{(1)}\left(\left(t_{1}+h_{2}\right)^{-}, t_{1}\right) A_{2} } \\
& =-\left(-A_{0}\right) A_{0}-\left[-X\left(\left(t_{1}+h_{1}\right)^{-}, t_{1}\right) A_{0}-X\left(\left(t_{1}+2 h_{1}\right)^{-}, t_{1}\right) A_{1}-X\left(\left(t_{1}+h_{1}+h_{2}\right)^{-}, t_{1}\right) A_{2}\right] A_{1} \\
& -\left[-X\left(\left(t_{1}+h_{2}\right)^{-}, t_{1}\right) A_{0}-X\left(\left(t_{1}+h_{2}+h_{1}\right)^{-}, t_{1}\right) A_{1}-X\left(\left(t_{1}+2 h_{2}\right)^{-}, t_{1}\right) A_{2}\right] A_{2} \\
& =A_{0}^{2}-\left[-0 . A_{0}-0 . A_{1}-0 . A_{2}\right] A_{1}-\left[-0 . A_{0}-0 . A_{1}-0 . A_{2}\right] A_{2}=A_{0}^{2}=(-1)^{2} A_{0}^{2}
\end{aligned}
$$

Thus (viii) is true for $k=2$. We assume that $X^{(k)}\left(t_{1}^{-}, t_{1}\right)=(-1)^{k} A_{0}^{k}$ for $2<k \leq n$, for some integer $n$. Then

$$
\begin{gathered}
X^{(n+1)}\left(t_{1}^{-}, t_{1}\right)=-\left.\left[\frac{\partial}{\partial \tau} X^{(n)}\left(\tau, t_{1}\right)\right]\right|_{\tau=t_{1}^{-}}=X^{(n)}\left(t_{1}^{-}, t_{1}\right) A_{0}-X^{(n)}\left(\left(t_{1}+h_{1}\right)^{-}, t_{1}\right) A_{1}-X^{(n)}\left(\left(t_{1}+h_{2}\right)^{-}, t_{1}\right) A_{2} \\
=(-1)(-1)^{n} A_{0}^{n} A_{0}-0 . A_{1}-0 . A_{2}=(-1)^{n+1} A_{0}^{n+1}
\end{gathered}
$$

Therefore $X^{(k)}\left(t_{1}^{-}, t_{1}\right)=(-1)^{k} A_{0}^{k}$, proving lemma (viii).

ix. $\quad X^{(k)}\left(t_{1}^{+}, t_{1}\right)=\lim _{t_{1}<\tau<t_{1}+h_{2}} X^{(k)}\left(\tau, t_{1}\right)=0$ (Since $\left.\tau>t_{1}\right)$

Therefore $X^{(k)}\left(t_{1}^{+}, t_{1}\right)=0$ proving lemma (ix).

x. $\quad \frac{\partial}{\partial \tau} X\left(\tau, t_{1}\right)=-X\left(\tau, t_{1}\right) A_{0}-X\left(\tau+h_{1}, t_{1}\right) A_{1}-X\left(\tau+h_{2}, t_{1}\right) A_{2}$

for $0<\tau<t_{1}, \tau \neq t_{1}-j h ; h=\max \left(h_{1}, h_{2}\right), j=0,1, \ldots$, where

$$
X(\tau, t)=\left\{\begin{array}{l}
I_{n}, \tau=t \\
0, \tau>t
\end{array}\right.
$$

Let $j$ be a non-negative number such that $t_{1}-j h>0$. Then we integrate the system $\frac{\partial}{\partial \tau} X\left(\tau, t_{1}\right)$, apply the above initial matrix function condition and the fundamental theorem of calculus (F.T.C) to get:

$$
\begin{aligned}
& \int_{0}^{\left(t_{1}-j h\right)^{-}} \frac{\partial}{\partial \tau}\left[X\left(\tau, t_{1}\right)\right] d \tau=X\left(\left(t_{1}-j h\right)^{-}, t_{1}\right)-X\left(0, t_{1}\right) \text { (by the F.T.C) } \\
& =-\int_{0}^{\left(t_{1}-j h\right)^{-}}\left[X\left(\tau, t_{1}\right) A_{0}+X\left(\tau+h_{1}, t_{1}\right) A_{1}+X\left(\tau+h_{2}, t_{1}\right) A_{2}\right] d \tau
\end{aligned}
$$

Similarly,

$$
X\left(\left(t_{1}-j h\right)^{+}, t_{1}\right)-X\left(0, t_{1}\right)=-\int_{0}^{\left(t_{1}-j h\right)^{+}}\left[X\left(\tau, t_{1}\right) A_{0}+X\left(\tau+h_{1}, t_{1}\right) A_{1}+X\left(\tau+h_{2}, t_{1}\right) A_{2}\right] d \tau
$$

Therefore

$$
\begin{aligned}
& X\left(\left(t_{1}-j h\right)^{-}, t_{1}\right)-X\left(\left(t_{1}-j h\right)^{+}, t_{1}\right) \\
& =-\int_{\left(t_{1}-j h\right)^{-}}^{\left(t_{1}-j h\right)^{+}}\left[X\left(\tau, t_{1}\right) A_{0}+X\left(\tau+h_{1}, t_{1}\right) A_{1}+X\left(\tau+h_{2}, t_{1}\right) A_{2}\right] d \tau=0
\end{aligned}
$$

Since $\tau \rightarrow X\left(\tau, t_{1}\right) A_{0}+X\left(\tau+h_{1}, t_{1}\right) A_{1}+X\left(\tau+h_{2}, t_{1}\right) A_{2}$ is bounded and integrable (being of bounded variation) and the fact that $\lim _{\varepsilon \rightarrow 0} \int_{a-\varepsilon}^{a+\varepsilon} f(t) d t=0$ for any bounded integrable function $f$. Therefore $\Delta X\left(t_{1}-j h, t_{1}\right)=0$, for $j \neq 0$. For $j=0$, we have: $\Delta X\left(t_{1}, t_{1}\right)=X\left(t_{1}^{-}, t_{1}\right)-X\left(t_{1}^{+}, t_{1}\right)=I_{n}-0=I_{n}$, which proves lemma (x). 


\section{Results}

3.1 Illustration of the Computation of the Determining Matrice $Q_{k}\left(j h_{1}\right) ; 0 \leq j \leq k, k \neq 0$ and $Q_{k}\left(j h_{1}\right) ; j>k \geq 1$ with respect to (1)

For notational optimality, we define: $\operatorname{SumProdPerm}\left[A_{0}\left(r_{0}\right), A_{1}\left(r_{1}\right), A_{2}\left(r_{2}\right)\right]$ to be the sum of the products of the permutations of $A_{0}, A_{1}$ and $A_{2}$ in which $A_{i}$ appears $r_{i}$ times, $i \in\{0,1,2\}$

3.1.1 Determining Matrices for $j=2, k \geq 2$

For $j=2, k=2$

$$
\begin{aligned}
& \qquad Q_{2}\left(2 h_{1}\right)=A_{0} Q_{1}\left(2 h_{1}\right)+A_{1} Q_{1}\left(h_{1}\right)+A_{2} Q_{1}\left(2 h_{1}-h_{2}\right) \\
& A_{0} A_{2}+A_{2} A_{0}+A_{1}^{2} ; h_{2}=2 h_{1} \\
& A_{1}^{2} ; \text { otherwise }
\end{aligned}
$$$$
=\left\{\begin{array}{l}
\text { SumProdPerm }\left[A_{0}(1), A_{1}(0), A_{2}(1)\right]+\operatorname{SumProdPerm}\left[A_{0}(0), A_{1}(2), A_{2}(0)\right] ; h_{2}=2 h_{1} \\
\text { SumProdPerm }\left[A_{0}(0), A_{1}(2), A_{2}(0)\right] ; \text { otherwise }
\end{array}\right.
$$

For $j=2, k=3$

$$
\begin{gathered}
Q_{3}\left(2 h_{1}\right)=A_{0} Q_{2}\left(2 h_{1}\right)+A_{1} Q_{2}\left(h_{1}\right)+A_{2} Q_{2}\left(2 h_{1}-h_{2}\right) \\
A_{0}^{2} A_{2}+A_{0} A_{1}^{2}+A_{0} A_{2} A_{0}+A_{1} A_{0} A_{1}+A_{1}^{2} A_{0}+A_{2} A_{0}^{2} ; h_{2}=2 h_{1} \\
A_{0} A_{1}^{2}+A_{1} A_{0} A_{1}+A_{1}^{2} A_{0} ; \text { otherwise }
\end{gathered}
$$$$
=\left\{\begin{array}{l}
\text { SumProdPerm }\left[A_{0}(2), A_{1}(0), A_{2}(1)\right]+\operatorname{SumProdPerm}\left[A_{0}(1), A_{1}(2), A_{2}(0)\right] ; h_{2}=2 h_{1} \\
\text { SumProdPerm }\left[A_{0}(1), A_{1}(2), A_{2}(0)\right] ; \text { otherwise }
\end{array}\right.
$$

For $j=2, k=4$

$$
\begin{gathered}
Q_{4}\left(2 h_{1}\right)=A_{0} Q_{3}\left(2 h_{1}\right)+A_{1} Q_{3}\left(h_{1}\right)+A_{2} Q_{3}\left(2 h_{1}-h_{2}\right) \\
=\left\{\begin{array}{l}
A_{0}^{3} A_{2}+A_{0}^{2} A_{1}^{2}+A_{0}^{2} A_{2} A_{0}+A_{0} A_{1} A_{0} A_{1}+A_{0} A_{1}^{2} A_{0}+A_{0} A_{2} A_{0}^{2}+A_{1} A_{0}^{2} A_{1}+A_{1} A_{0} A_{1} A_{0}+A_{1}^{2} A_{0}^{2}+A_{2} A_{0}^{3} ; h_{2}=2 h_{1} \\
A_{0}^{2} A_{1}^{2}+A_{0} A_{1} A_{0} A_{1}+A_{0} A_{1}^{2} A_{0}+A_{1} A_{0}^{2} A_{1}+A_{1} A_{0} A_{1} A_{0}+A_{1}^{2} A_{0}^{2} ; \text { otherwise }
\end{array}\right. \\
=\left\{\begin{array}{l}
\text { SumProdPerm }\left[A_{0}(3), A_{1}(0), A_{2}(1)\right]+\operatorname{SumProdPerm}\left[A_{0}(2), A_{1}(2), A_{2}(0)\right] ; h_{2}=2 h_{1} \\
\text { SumProdPerm }\left[A_{0}(2), A_{1}(2), A_{2}(0)\right] ; \text { otherwise }
\end{array}\right.
\end{gathered}
$$

For $j=2, k=5$

$$
Q_{5}\left(2 h_{1}\right)=A_{0} Q_{4}\left(2 h_{1}\right)+A_{1} Q_{4}\left(h_{1}\right)+A_{2} Q_{4}\left(2 h_{1}-h_{2}\right)
$$




$$
\begin{aligned}
& \left(A_{0}{ }^{4} A_{2}+A_{0}^{3} A_{1}^{2}+A_{0}^{3} A_{2} A_{0}+A_{0}{ }^{2} A_{1} A_{0} A_{1}+A_{0}{ }^{2} A_{1}{ }^{2} A_{0}+A_{0}{ }^{2} A_{2} A_{0}{ }^{2}+A_{0} A_{1} A_{0}{ }^{2} A_{1}+A_{0} A_{1} A_{0} A_{1} A_{0}\right. \\
& +A_{0} A_{1}^{2} A_{0}^{2}+A_{0} A_{2} A_{0}^{3}+A_{1} A_{0}^{3} A_{1}+A_{1} A_{0}^{2} A_{1} A_{0}+A_{1} A_{0} A_{1} A_{0}^{2}+A_{1}^{2} A_{0}^{3}+A_{2} A_{0}^{4} ; h_{2}=2 h_{1} \\
& =\{ \\
& A_{0}^{3} A_{1}^{2}+A_{0}^{2} A_{1} A_{0} A_{1}+A_{0}^{2} A_{1}^{2} A_{0}+A_{0} A_{1} A_{0}^{2} A_{1}+A_{0} A_{1} A_{0} A_{1} A_{0}+A_{0} A_{1}^{2} A_{0}^{2}+A_{1} A_{0}^{3} A_{1}+A_{1} A_{0}{ }^{2} A_{1} A_{0} \\
& +A_{1} A_{0} A_{1} A_{0}^{2}+A_{1}^{2} A_{0}^{3} \text {; otherwise } \\
& =\left\{\begin{array}{l}
\text { SumProdPerm }\left[A_{0}(4), A_{1}(0), A_{2}(1)\right]+\operatorname{SumProdPerm}\left[A_{0}(3), A_{1}(2), A_{2}(0)\right] ; h_{2}=2 h_{1} \\
\text { SumProdPerm }\left[A_{0}(3), A_{1}(2), A_{2}(0)\right] ; \text { otherwise }
\end{array}\right.
\end{aligned}
$$

SumProdPerm $\left[A_{0}(3), A_{1}(2), A_{2}(0)\right]$; otherwise

Emerging pattern of $Q_{k}\left(j h_{1}\right)$ for $j=2, k \geq 2$ :

$$
=\left\{\begin{array}{l}
\sum_{r=0}^{1} \operatorname{SumProdPerm}\left[A_{0}(r+k-2), A_{1}(2-2 r), A_{2}(r)\right] ; h_{2}=2 h_{1} \\
\text { SumProdPerm }\left[A_{0}(k-2), A_{1}(2), A_{2}(0)\right] ; \text { otherwise }
\end{array}\right.
$$

3.1.2 Determining Matrices for $j=3, k \geq 2$

For $j=3, k=2$

$$
\begin{aligned}
& \quad Q_{2}\left(3 h_{1}\right)=A_{0} Q_{1}\left(3 h_{1}\right)+A_{1} Q_{1}\left(2 h_{1}\right)+A_{2} Q_{1}\left(3 h_{1}-h_{2}\right) \\
= & \left\{\begin{array}{l}
A_{1} A_{2}+A_{2} A_{1} ; h_{2}=2 h_{1} \\
A_{0} A_{2}+A_{2} A_{0} ; h_{2}=3 h_{1} \\
0 ; \text { otherwise }
\end{array}\right. \\
= & \left\{\begin{array}{l}
\text { SumProdPerm }\left[A_{0}(0), A_{1}(1), A_{2}(1)\right] ; h_{2}=2 h_{1} \\
\text { SumProdPerm }\left[A_{0}(1), A_{1}(0), A_{2}(1)\right] ; h_{2}=3 h_{1} \\
0 ; \text { otherwise }
\end{array}\right.
\end{aligned}
$$

For $j=3, k=3$

$$
\begin{gathered}
Q_{3}\left(3 h_{1}\right)=A_{0} Q_{2}\left(3 h_{1}\right)+A_{1} Q_{2}\left(2 h_{1}\right)+A_{2} Q_{2}\left(3 h_{1}-h_{2}\right) \\
=\left\{\begin{array}{l}
A_{0} A_{1} A_{2}+A_{0} A_{2} A_{1}+A_{1} A_{0} A_{2}+A_{1}^{3}+A_{1} A_{2} A_{0}+A_{2} A_{0} A_{1}+A_{2} A_{1} A_{0} ; h_{2}=2 h_{1} \\
A_{0}^{2} A_{2}+A_{0} A_{2} A_{0}+A_{1}^{3}+A_{2} A_{0}^{2} ; h_{2}=3 h_{1} \\
A_{1}^{3}
\end{array}\right.
\end{gathered}
$$




$$
=\left\{\begin{array}{l}
\operatorname{SumProdPerm}\left[A_{0}(1), A_{1}(1), A_{2}(1)\right]+\operatorname{SumProdPerm}\left[A_{0}(0), A_{1}(3), A_{2}(0)\right] ; h_{2}=2 h_{1} \\
\operatorname{SumProdPerm}\left[A_{0}(2), A_{1}(0), A_{2}(1)\right]+\operatorname{SumProdPerm}\left[A_{0}(0), A_{1}(3), A_{2}(0)\right] ; h_{2}=3 h_{1} \\
\text { SumProdPerm }\left[A_{0}(0), A_{1}(3), A_{2}(0)\right] ; \text { otherwise }
\end{array}\right.
$$$$
\text { For } j=3, k=4
$$$$
\begin{gathered}
\quad Q_{4}\left(3 h_{1}\right)=A_{0} Q_{3}\left(3 h_{1}\right)+A_{1} Q_{3}\left(2 h_{1}\right)+A_{2} Q_{3}\left(3 h_{1}-h_{2}\right) \\
=\left\{\begin{array}{l}
A_{0}^{2} A_{1} A_{2}+A_{0}^{2} A_{2} A_{1}+A_{0} A_{1} A_{0} A_{2}+A_{0} A_{1}^{3}+A_{0} A_{1} A_{2} A_{0}+A_{0} A_{2} A_{0} A_{1}+A_{0} A_{2} A_{1} A_{0}+A_{1} A_{0}^{2} A_{2}+A_{1} A_{0} A_{1}^{2} \\
+A_{1} A_{0} A_{2} A_{0}+A_{1}^{2} A_{0} A_{1}+A_{1}^{3} A_{0}+A_{1} A_{2} A_{0}^{2}+A_{2} A_{0}^{2} A_{1}+A_{2} A_{0} A_{1} A_{0}+A_{2} A_{1} A_{0}^{2} ; h_{2}=2 h_{1} \\
A_{0}^{3} A_{2}+A_{0}^{2} A_{2} A_{0}+A_{0} A_{1}^{3}+A_{0} A_{2} A_{0}^{2}+A_{1} A_{0} A_{1}^{2}+A_{1}^{2} A_{0} A_{1}+A_{1}^{3} A_{0}+A_{2} A_{0}^{3} ; h_{2}=3 h_{1} \\
A_{0} A_{1}^{3}+A_{1} A_{0} A_{1}^{2}+A_{1}^{2} A_{0} A_{1}+A_{1}^{3} A_{0} ; \text { otherwise }
\end{array}\right.
\end{gathered}
$$$$
=\left\{\begin{array}{l}
\text { SumProdPerm }\left[A_{0}(2), A_{1}(1), A_{2}(1)\right]+\operatorname{SumProdPerm}\left[A_{0}(1), A_{1}(3), A_{2}(0)\right] ; h_{2}=2 h_{1} \\
\operatorname{SumProdPerm}\left[A_{0}(3), A_{1}(0), A_{2}(1)\right]+\operatorname{SumProdPerm}\left[A_{0}(1), A_{1}(3), A_{2}(0)\right] ; h_{2}=3 h_{1}
\end{array}\right.
$$$$
\text { SumProdPerm }\left[A_{0}(1), A_{1}(3), A_{2}(0)\right] \text {; otherwise }
$$

Emerging pattern of $Q_{k}\left(j h_{1}\right)$ for $j=3, k \geq 3$

$$
=\left\{\begin{array}{l}
\sum_{r=0}^{1} \text { SumProdPerm }\left[A_{0}(r+k-3), A_{1}(3-2 r), A_{2}(r)\right] ; h_{2}=2 h_{1} \\
\sum_{r=0}^{1} \operatorname{SumProdPerm}\left[A_{0}(2 r+k-3), A_{1}(3-3 r), A_{2}(r)\right] ; h_{2}=3 h_{1} \\
\text { SumProdPerm }\left[A_{0}(k-3), A_{1}(3), A_{2}(0)\right] ; \text { otherwise }
\end{array}\right.
$$

3.1.3 Determining Matrices for $j=4, k \geq 2$

For $j=4, k=2$

$$
Q_{2}\left(4 h_{1}\right)=A_{0} Q_{1}\left(4 h_{1}\right)+A_{1} Q_{1}\left(3 h_{1}\right)+A_{2} Q_{1}\left(4 h_{1}-h_{2}\right)
$$




$$
\begin{aligned}
& =\left\{\begin{array}{l}
A_{2}^{2} ; h_{2}=2 h_{1} \\
A_{1} A_{2}+A_{2} A_{1} ; h_{2}=3 h_{1} \\
A_{0} A_{2}+A_{2} A_{0} ; h_{2}=4 h_{1}
\end{array}\right. \\
& 0 \text {; otherwise } \\
& =\left\{\begin{array}{l}
\text { SumProdPerm }\left[A_{0}(0), A_{1}(0), A_{2}(2)\right] ; h_{2}=2 h_{1} \\
\text { SumProdPerm }\left[A_{0}(0), A_{1}(1), A_{2}(1)\right] ; h_{2}=3 h_{1} \\
\text { SumProdPerm }\left[A_{0}(1), A_{1}(0), A_{2}(1)\right] ; h_{2}=4 h_{1}
\end{array}\right. \\
& 0 \text {; otherwise }
\end{aligned}
$$

For $j=4, k=3$

$$
\begin{aligned}
& Q_{3}\left(4 h_{1}\right)=A_{0} Q_{2}\left(4 h_{1}\right)+A_{1} Q_{2}\left(3 h_{1}\right)+A_{2} Q_{2}\left(4 h_{1}-h_{2}\right) \\
& =\left\{\begin{array}{l}
A_{0} A_{2}^{2}+A_{1}^{2} A_{2}+A_{1} A_{2} A_{1}+A_{2} A_{0} A_{2}+A_{2} A_{1}^{2}+A_{2}^{2} A_{0} ; h_{2}=2 h_{1} \\
A_{0} A_{1} A_{2}+A_{0} A_{2} A_{1}+A_{1} A_{0} A_{2}+A_{1} A_{2} A_{0}+A_{2} A_{0} A_{1}+A_{2} A_{1} A_{0} ; h_{2}=3 h_{1} \\
A_{0}^{2} A_{2}+A_{0} A_{2} A_{0}+A_{2} A_{0}^{2} ; h_{2}=4 h_{1}
\end{array}\right. \\
& 0 \text {; otherwise } \\
& \text { SumProdPerm }\left[A_{0}(1), A_{1}(0), A_{2}(2)\right]+\operatorname{SumProdPerm}\left[A_{0}(0), A_{1}(2), A_{2}(1)\right] ; h_{2}=2 h_{1} \\
& \text { SumProdPerm }\left[A_{0}(1), A_{1}(1), A_{2}(1)\right] ; h_{2}=3 h_{1} \\
& =\{ \\
& \text { SumProdPerm }\left[A_{0}(2), A_{1}(0), A_{2}(1)\right] ; h_{2}=4 h_{1} \\
& 0 \text {; otherwise } \\
& \text { For } j=4, k=4
\end{aligned}
$$

$$
Q_{4}\left(4 h_{1}\right)=A_{0} Q_{3}\left(4 h_{1}\right)+A_{1} Q_{3}\left(3 h_{1}\right)+A_{2} Q_{3}\left(4 h_{1}-h_{2}\right)
$$




$$
\begin{aligned}
& A_{0}^{2} A_{2}^{2}+A_{0} A_{1}^{2} A_{2}+A_{0} A_{1} A_{2} A_{1}+A_{0} A_{2} A_{0} A_{2}+A_{0} A_{2} A_{1}^{2}+A_{0} A_{2}^{2} A_{0}+A_{1} A_{0} A_{1} A_{2}+A_{1}^{2} A_{0} A_{2} \\
& +A_{1}^{4}+A_{1}^{2} A_{2} A_{0}+A_{1} A_{2} A_{0} A_{1}+A_{1} A_{2} A_{1} A_{0}+A_{2} A_{0}^{2} A_{2}+A_{2} A_{0} A_{1}^{2}+A_{2} A_{0} A_{2} A_{0}+A_{2} A_{1} A_{0} A_{1} \\
& +A_{2} A_{1}^{2} A_{0}+A_{2}^{2} A_{0}^{2} ; h_{2}=2 h_{1} \\
& =\left\{\begin{array}{l}
A_{0}^{2} A_{1} A_{2}+A_{0}^{2} A_{2} A_{1}+A_{0} A_{1} A_{0} A_{2}+A_{0} A_{1} A_{2} A_{0}+A_{0} A_{2} A_{0} A_{1}+A_{0} A_{2} A_{1} A_{0}+A_{1} A_{0}^{2} A_{2}+A_{1} A_{0} A_{2} A_{0} \\
+A_{1}^{4}+A_{1} A_{2} A_{0}^{2} A_{2} A_{0}^{2} A_{1} A_{2} A_{0} A_{1} A_{0} A_{2} A_{1} A_{0}^{2}
\end{array}\right. \\
& +A_{1}^{4}+A_{1} A_{2} A_{0}^{2}+A_{2} A_{0}^{2} A_{1}+A_{2} A_{0} A_{1} A_{0}+A_{2} A_{1} A_{0}^{2} ; h_{2}=3 h_{1} \\
& A_{0}^{3} A_{2}+A_{0}^{2} A_{2} A_{0}+A_{0} A_{2} A_{0}^{2}+A_{2} A_{0}^{3}+A_{1}^{4} ; h_{2}=4 h_{1} \\
& A_{1}^{4} ; \text { otherwise } \\
& \text { SumProdPerm }\left[A_{0}(2), A_{1}(0), A_{2}(2)\right]+\operatorname{SumProdPerm}\left[A_{0}(1), A_{1}(2), A_{2}(1)\right] \\
& + \text { SumProdPerm }\left[A_{0}(0), A_{1}(4), A_{2}(0)\right] ; h_{2}=2 h_{1} \\
& =\left\{\operatorname{SumProdPerm}\left[A_{0}(2), A_{1}(1), A_{2}(1)\right]+\operatorname{SumProdPerm}\left[A_{0}(0), A_{1}(4), A_{2}(0)\right] ; h_{2}=3 h_{1}\right. \\
& \operatorname{SumProdPerm}\left[A_{0}(3), A_{1}(0), A_{2}(1)\right]+\operatorname{SumProdPerm}\left[A_{0}(0), A_{1}(4), A_{2}(0)\right] ; h_{2}=4 h_{1} \\
& \text { SumProdPerm }\left[A_{0}(0), A_{1}(4), A_{2}(0)\right] \text {; otherwise }
\end{aligned}
$$$$
\text { For } j=4, k=5
$$$$
Q_{5}\left(4 h_{1}\right)=A_{0} Q_{4}\left(4 h_{1}\right)+A_{1} Q_{4}\left(3 h_{1}\right)+A_{2} Q_{4}\left(4 h_{1}-h_{2}\right)
$$ 


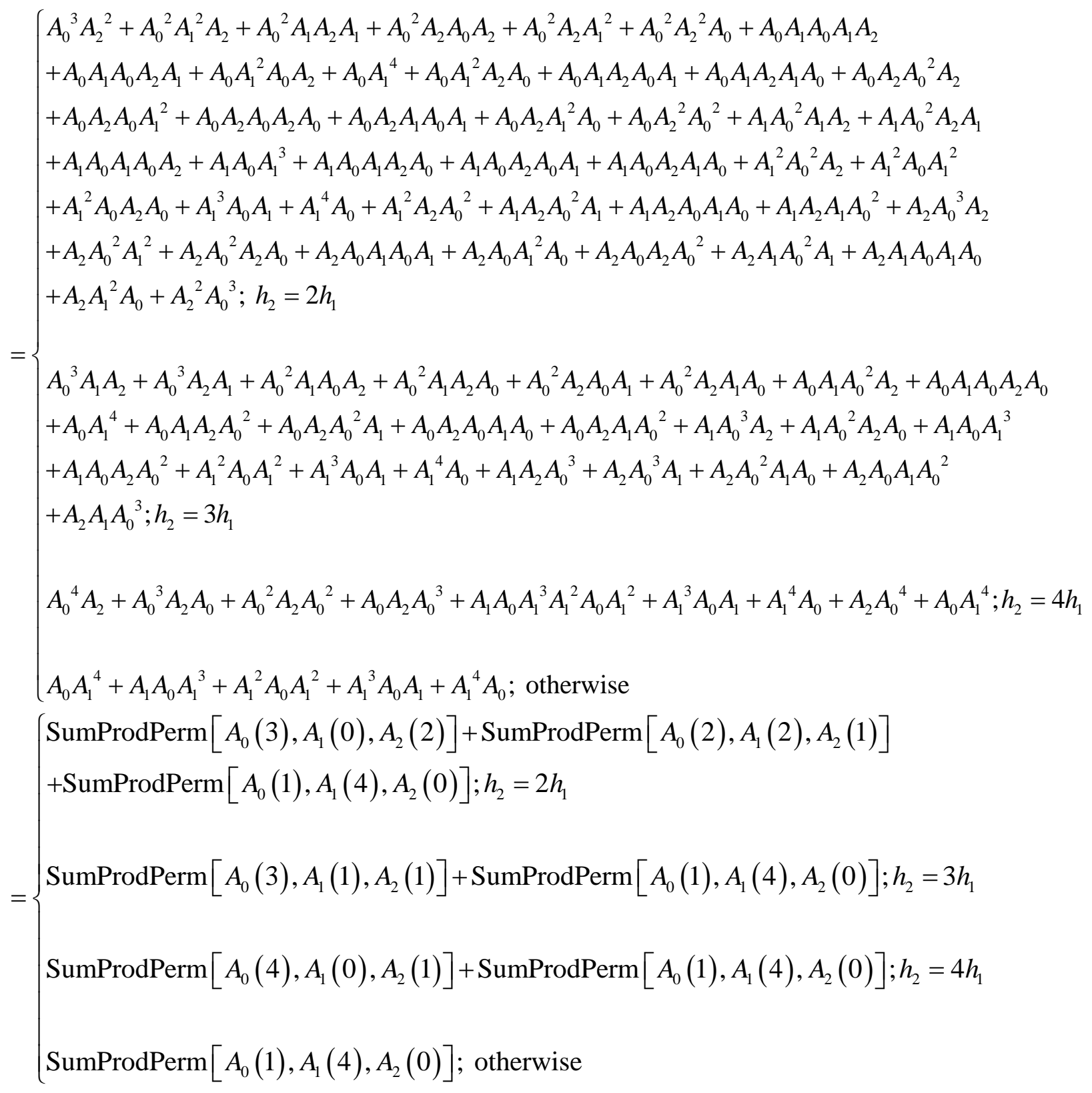

Emerging pattern for $j=4, k \geq 4$ 


$$
Q_{k}\left(4 h_{1}\right)=\left\{\begin{array}{l}
\sum_{r=0}^{2} \operatorname{SumProdPerm}\left[A_{0}(r+k-4), A_{1}(4-2 r), A_{2}(r)\right] ; h_{2}=2 h_{1} \\
\sum_{r=0}^{1} \operatorname{SumProdPerm}\left[A_{0}(2 r+k-4), A_{1}(4-3 r), A_{2}(r)\right] ; h_{2}=3 h_{1} \\
\sum_{r=0}^{1} \operatorname{SumProdPerm}\left[A_{0}(3 r+k-4), A_{1}(4-4 r), A_{2}(r)\right] ; h_{2}=4 h_{1} \\
\text { SumProdPerm }\left[A_{0}(k-4), A_{1}(4), A_{2}(0)\right] ; \text { otherwise }
\end{array}\right.
$$

3.1.4 Determining Matrices for $j=5, k \geq 2$

For $j=5, k=2$

$$
\begin{aligned}
& Q_{2}\left(5 h_{1}\right)=A_{0} Q_{1}\left(5 h_{1}\right)+A_{1} Q_{1}\left(4 h_{1}\right)+A_{2} Q_{1}\left(5 h_{1}-h_{2}\right) \\
& 0 ; h_{2}=2 h_{1} \\
& 0 ; h_{2}=3 h_{1} \\
& A_{1} A_{2}+A_{2} A_{1} ; h_{2}=4 h_{1} \\
& A_{0} A_{2}+A_{2} A_{0} ; h_{2}=5 h_{1} \\
& 0 ; \text { otherwise } \\
& 0 ; h_{2}=2 h_{1} \\
& 0 ; h_{2}=3 h_{1} \\
& \text { SumProdPerm }\left[A_{0}(0), A_{1}(1), A_{2}(1)\right] ; h_{2}=4 h_{1} \\
& \text { SumProdPerm }\left[A_{0}(1), A_{1}(0), A_{2}(1)\right] ; h_{2}=5 h_{1} \\
& 0 ; \text { otherwise }
\end{aligned}
$$$$
\text { For } j=5, k=3
$$$$
Q_{3}\left(5 h_{1}\right)=A_{0} Q_{2}\left(5 h_{1}\right)+A_{1} Q_{2}\left(4 h_{1}\right)+A_{2} Q_{2}\left(5 h_{1}-h_{2}\right)
$$ 


$$
=\left\{\begin{array}{l}
A_{1} A_{2}^{2}+A_{2} A_{1} A_{2}+A_{2}^{2} A_{1} ; h_{2}=2 h_{1} \\
A_{1}^{2} A_{2}+A_{1} A_{2} A_{1}+A_{2} A_{1}^{2} ; h_{2}=3 h_{1} \\
A_{0} A_{1} A_{2}+A_{0} A_{2} A_{1}+A_{1} A_{0} A_{2}+A_{1} A_{2} A_{0}+A_{2} A_{0} A_{1}+A_{2} A_{1} A_{0} ; h_{2}=4 h_{1} \\
A_{0}^{2} A_{2}+A_{0} A_{2} A_{0}+A_{2} A_{0}^{2} ; h_{2}=5 h_{1} \\
0 ; \text { otherwise }
\end{array}\right.
$$

$$
=\left\{\begin{array}{l}
\text { SumProdPerm }\left[A_{0}(0), A_{1}(1), A_{2}(2)\right] ; h_{2}=2 h_{1} \\
\text { SumProdPerm }\left[A_{0}(0), A_{1}(2), A_{2}(1)\right] ; h_{2}=3 h_{1} \\
\text { SumProdPerm }\left[A_{0}(1), A_{1}(1), A_{2}(1)\right] ; h_{2}=4 h_{1} \\
\text { SumProdPerm }\left[A_{0}(2), A_{1}(0), A_{2}(1)\right] ; h_{2}=5 h_{1}
\end{array}\right.
$$

0 ; otherwise

For $j=5, k=4$

$$
\begin{gathered}
\quad Q_{4}\left(5 h_{1}\right)=A_{0} Q_{3}\left(5 h_{1}\right)+A_{1} Q_{3}\left(4 h_{1}\right)+A_{2} Q_{3}\left(5 h_{1}-h_{2}\right) \\
+A_{2} A_{0} A_{1} A_{2}+A_{2} A_{0} A_{2} A_{1}+A_{2} A_{1} A_{0} A_{1}+A_{2} A_{1}^{2} A_{0}+A_{2}^{2} A_{0} A_{2}+A_{2}^{2} A_{1} A_{0} ; h_{2}=2 h_{1} \\
A_{0} A_{1}^{2} A_{2}+A_{0} A_{1} A_{2} A_{1}+A_{0} A_{2} A_{1}^{2}+A_{1} A_{0} A_{1} A_{2}+A_{1} A_{0} A_{2} A_{1}+A_{1}^{2} A_{0} A_{2}+A_{1}^{2} A_{2} A_{0}+A_{1} A_{2} A_{0} A_{1}+A_{1} A_{2} A_{1} A_{0} \\
+A_{2} A_{0} A_{1}^{2}+A_{2} A_{1} A_{0} A_{1}+A_{2} A_{1}^{2} A_{0} ; h_{2}=3 h_{1} \\
A_{0}^{2} A_{1} A_{2}+A_{0}^{2} A_{2} A_{1}+A_{0} A_{1} A_{0} A_{2}+A_{0} A_{1} A_{2} A_{0}+A_{0} A_{2} A_{0} A_{1}+A_{0} A_{2} A_{1} A_{0}+A_{1} A_{0}^{2} A_{2}+A_{1} A_{0} A_{2} A_{0}+A_{1} A_{2} A_{0}^{2} \\
+A_{2} A_{0}^{2} A_{1}+A_{2} A_{0} A_{1} A_{0}+A_{2} A_{1} A_{0}^{2} ; h_{2}=4 h_{1} \\
A_{0}^{3} A_{2}+A_{0}^{2} A_{2} A_{0}+A_{0} A_{2} A_{0}^{2}+A_{2} A_{0}^{3} ; h_{2}=5 h_{1} \\
0 ; \text { otherwise }
\end{gathered}
$$




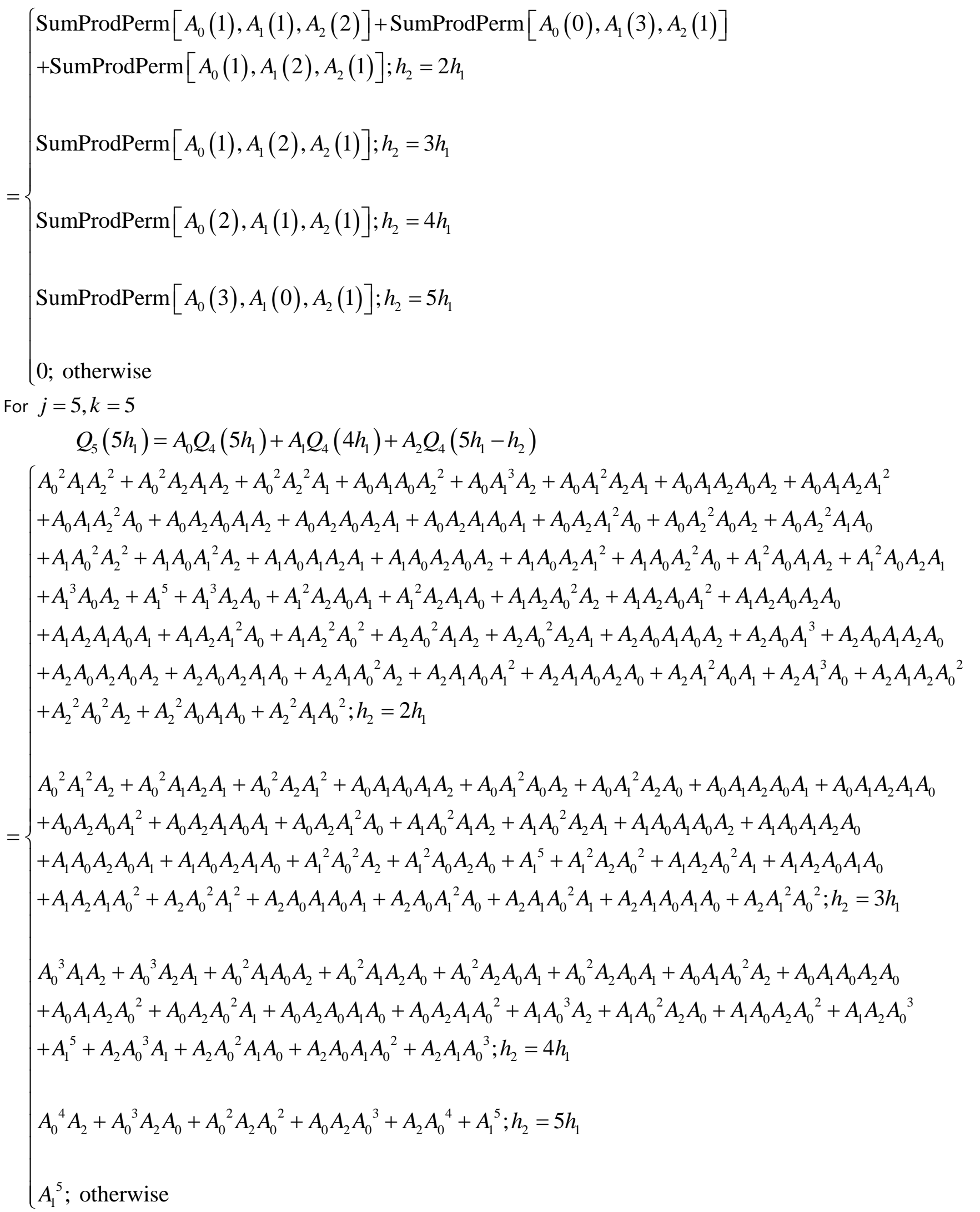




$$
\begin{aligned}
& =\left\{\begin{array}{l}
\operatorname{SumProdPerm}\left[A_{0}(2), A_{1}(1), A_{2}(2)\right]+\operatorname{SumProdPerm}\left[A_{0}(1), A_{1}(3), A_{2}(1)\right] \\
+\operatorname{SumProdPerm}\left[A_{0}(0), A_{1}(5), A_{2}(0)\right] ; h_{2}=2 h_{1} \\
\operatorname{SumProdPerm}\left[A_{0}(2), A_{1}(2), A_{2}(1)\right]+\operatorname{SumProdPerm}\left[A_{0}(0), A_{1}(5), A_{2}(0)\right] ; h_{2}=3 h_{1} \\
\operatorname{SumProdPerm}\left[A_{0}(3), A_{1}(1), A_{2}(1)\right]+\operatorname{SumProdPerm}\left[A_{0}(0), A_{1}(5), A_{2}(0)\right] ; h_{2}=4 h_{1} \\
\operatorname{SumProdPerm}\left[A_{0}(4), A_{1}(0), A_{2}(1)\right]+\operatorname{SumProdPerm}\left[A_{0}(0), A_{1}(5), A_{2}(0)\right] ; h_{2}=5 h_{1} \\
\text { SumProdPerm }\left[A_{0}(0), A_{1}(5), A_{2}(0)\right] ; \text { otherwise }
\end{array}\right. \\
& \text { For } j=5, k=6
\end{aligned}
$$$$
Q_{6}\left(5 h_{1}\right)=A_{0} Q_{5}\left(5 h_{1}\right)+A_{1} Q_{5}\left(4 h_{1}\right)+A_{2} Q_{5}\left(5 h_{1}-h_{2}\right)
$$$$
\text { SumProdPerm }\left[A_{0}(3), A_{1}(1), A_{2}(2)\right]+\operatorname{SumProdPerm}\left[A_{0}(2), A_{1}(3), A_{2}(1)\right]
$$$$
+ \text { SumProdPerm }\left[A_{0}(1), A_{1}(5), A_{2}(0)\right] ; h_{2}=2 h_{1}
$$$$
\text { SumProdPerm }\left[A_{0}(3), A_{1}(2), A_{2}(1)\right]+\operatorname{SumProdPerm}\left[A_{0}(1), A_{1}(5), A_{2}(0)\right] ; h_{2}=3 h_{1}
$$$$
=\left\{\begin{array}{l}
\text { SumProdPerm }\left[A_{0}(4), A_{1}(1), A_{2}(1)\right]+\operatorname{SumProdPerm}\left[A_{0}(1), A_{1}(5), A_{2}(0)\right] ; h_{2}=4 h_{1}
\end{array}\right.
$$$$
\operatorname{SumProdPerm}\left[A_{0}(5), A_{1}(0), A_{2}(1)\right]+\operatorname{SumProdPerm}\left[A_{0}(1), A_{1}(5), A_{2}(0)\right] ; h_{2}=5 h_{1}
$$$$
\text { SumProdPerm }\left[A_{0}(1), A_{1}(5), A_{2}(0)\right] \text {; otherwise }
$$

Emerging pattern for $j=5, k \geq 5$ 


$$
Q_{k}\left(5 h_{1}\right)=\left\{\begin{array}{l}
\sum_{r=0}^{2} \operatorname{SumProdPerm}\left[A_{0}(r+k-5), A_{1}(5-2 r), A_{2}(r)\right] ; h_{2}=2 h_{1} \\
\sum_{r=0}^{1} \operatorname{SumProdPerm}\left[A_{0}(2 r+k-5), A_{1}(5-3 r), A_{2}(r)\right] ; h_{2}=3 h_{1} \\
r=0 \\
\sum_{r=0}^{1} \operatorname{SumProdPerm}\left[A_{0}(4 r+k-5), A_{1}(5-5 r), A_{2}(r)\right] ; h_{2}=5 h_{1} \\
\text { SumProdPerm }\left[A_{0}(k-5), A_{1}(5), A_{2}(0)\right] ; \text { otherwise }
\end{array}\right.
$$

3.1.5 Determining Matrices for $j=6, k \geq 2$

For $j=6, k=2$

$Q_{2}\left(6 h_{1}\right)=A_{0} Q_{1}\left(6 h_{1}\right)+A_{1} Q_{1}\left(5 h_{1}\right)+A_{2} Q_{1}\left(6 h_{1}-h_{2}\right)$

$\left\{\begin{array}{l}0 ; h_{2}=2 h_{1} \\ \text { SumProdPerm }\left[A_{0}(0), A_{1}(0), A_{2}(2)\right] ; h_{2}=3 h_{1} \\ 0 ; h_{2}=4 h_{1} \\ \text { SumProdPerm }\left[A_{0}(0), A_{1}(1), A_{2}(1)\right] ; h_{2}=5 h_{1} \\ \text { SumProdPerm }\left[A_{0}(1), A_{1}(0), A_{2}(1)\right] ; h_{2}=6 h_{1} \\ 0 ; \text { otherwise }\end{array}\right.$

For $j=6, k=3$

$Q_{3}\left(6 h_{1}\right)=A_{0} Q_{2}\left(6 h_{1}\right)+A_{1} Q_{2}\left(5 h_{1}\right)+A_{2} Q_{2}\left(6 h_{1}-h_{2}\right)$ 


$$
=\left\{\begin{array}{l}
\text { SumProdPerm }\left[A_{0}(0), A_{1}(0), A_{2}(3)\right] ; h_{2}=2 h_{1} \\
\text { SumProdPerm }\left[A_{0}(1), A_{1}(0), A_{2}(2)\right] ; h_{2}=3 h_{1} \\
\text { SumProdPerm }\left[A_{0}(0), A_{1}(2), A_{2}(1)\right] ; h_{2}=4 h_{1} \\
\text { SumProdPerm }\left[A_{0}(1), A_{1}(1), A_{2}(1)\right] ; h_{2}=5 h_{1} \\
\text { SumProdPerm }\left[A_{0}(2), A_{1}(0), A_{2}(1)\right] ; h_{2}=6 h_{1} \\
0 ; \text { otherwise }
\end{array}\right.
$$

For $j=6, k=4$

$$
Q_{4}\left(6 h_{1}\right)=A_{0} Q_{3}\left(6 h_{1}\right)+A_{1} Q_{3}\left(5 h_{1}\right)+A_{2} Q_{3}\left(6 h_{1}-h_{2}\right)
$$

$$
=\left\{\begin{array}{l}
\operatorname{SumProdPerm}\left[A_{0}(1), A_{1}(0), A_{2}(3)\right]+\operatorname{SumProdPerm}\left[A_{0}(0), A_{1}(2), A_{2}(2)\right] ; h_{2}=2 h_{1} \\
\operatorname{SumProdPerm}\left[A_{0}(2), A_{1}(0), A_{2}(2)\right]+\operatorname{SumProdPerm}\left[A_{0}(0), A_{1}(3), A_{2}(1)\right] ; h_{2}=3 h_{1} \\
\operatorname{SumProdPerm}\left[A_{0}(1), A_{1}(2), A_{2}(1)\right] ; h_{2}=4 h_{1} \\
\text { SumProdPerm }\left[A_{0}(2), A_{1}(1), A_{2}(1)\right] ; h_{2}=5 h_{1} \\
\text { SumProdPerm }\left[A_{0}(3), A_{1}(0), A_{2}(1)\right] ; h_{2}=6 h_{1} \\
0 ; \text { otherwise }
\end{array}\right.
$$

For $j=6, k=5$

$$
Q_{5}\left(6 h_{1}\right)=A_{0} Q_{4}\left(6 h_{1}\right)+A_{1} Q_{4}\left(5 h_{1}\right)+A_{2} Q_{4}\left(6 h_{1}-h_{2}\right)
$$




$$
\begin{aligned}
& \text { SumProdPerm }\left[A_{0}(2), A_{1}(0), A_{2}(3)\right]+\operatorname{SumProdPerm}\left[A_{0}(1), A_{1}(2), A_{2}(2)\right] \\
& + \text { SumProdPerm }\left[A_{0}(0), A_{1}(4), A_{2}(1)\right] ; h_{2}=2 h_{1} \\
& \operatorname{SumProdPerm}\left[A_{0}(3), A_{1}(0), A_{2}(2)\right]+\operatorname{SumProdPerm}\left[A_{0}(1), A_{1}(3), A_{2}(1)\right] ; h_{2}=3 h_{1} \\
& =\left\{\text { SumProdPerm }\left[A_{0}(2), A_{1}(2), A_{2}(1)\right] ; h_{2}=4 h_{1}\right. \\
& \text { SumProdPerm }\left[A_{0}(3), A_{1}(1), A_{2}(1)\right] ; h_{2}=5 h_{1} \\
& \text { SumProdPerm }\left[A_{0}(4), A_{1}(0), A_{2}(1)\right] ; h_{2}=6 h_{1} \\
& 0 \text {; otherwise } \\
& \text { For } j=6, k=6 \\
& Q_{6}\left(6 h_{1}\right)=A_{0} Q_{5}\left(6 h_{1}\right)+A_{1} Q_{5}\left(5 h_{1}\right)+A_{2} Q_{5}\left(6 h_{1}-h_{2}\right) \\
& \text { SumProdPerm }\left[A_{0}(3), A_{1}(0), A_{2}(3)\right]+\operatorname{SumProdPerm}\left[A_{0}(2), A_{1}(2), A_{2}(2)\right] \\
& + \text { SumProdPerm }\left[A_{0}(1), A_{1}(4), A_{2}(1)\right]+\operatorname{SumProdPerm}\left[A_{0}(0), A_{1}(6), A_{2}(0)\right] ; h_{2}=2 h_{1} \\
& \text { SumProdPerm }\left[A_{0}(4), A_{1}(0), A_{2}(2)\right]+\operatorname{SumProdPerm}\left[A_{0}(2), A_{1}(3), A_{2}(1)\right] \\
& + \text { SumProdPerm }\left[A_{0}(0), A_{1}(6), A_{2}(0)\right] ; h_{2}=3 h_{1} \\
& =\left\{\operatorname{SumProdPerm}\left[A_{0}(3), A_{1}(2), A_{2}(1)\right]+\operatorname{SumProdPerm}\left[A_{0}(0), A_{1}(6), A_{2}(0)\right] ; h_{2}=4 h_{1}\right. \\
& \text { SumProdPerm }\left[A_{0}(4), A_{1}(1), A_{2}(1)\right]+\operatorname{SumProdPerm}\left[A_{0}(0), A_{1}(6), A_{2}(0)\right] ; h_{2}=5 h_{1} \\
& \text { SumProdPerm }\left[A_{0}(5), A_{1}(0), A_{2}(1)\right]+\operatorname{SumProdPerm}\left[A_{0}(0), A_{1}(6), A_{2}(0)\right] ; h_{2}=6 h_{1} \\
& \text { SumProdPerm }\left[A_{0}(0), A_{1}(6), A_{2}(0)\right] \text {; otherwise }
\end{aligned}
$$$$
\text { For } j=6, k=7
$$$$
Q_{7}\left(6 h_{1}\right)=A_{0} Q_{6}\left(6 h_{1}\right)+A_{1} Q_{6}\left(5 h_{1}\right)+A_{2} Q_{6}\left(6 h_{1}-h_{2}\right)
$$ 


$$
\begin{aligned}
& \text { SumProdPerm }\left[A_{0}(4), A_{1}(0), A_{2}(3)\right]+\operatorname{SumProdPerm}\left[A_{0}(3), A_{1}(2), A_{2}(2)\right] \\
& + \text { SumProdPerm }\left[A_{0}(2), A_{1}(4), A_{2}(1)\right]+\operatorname{SumProdPerm}\left[A_{0}(1), A_{1}(6), A_{2}(0)\right] ; h_{2}=2 h_{1} \\
& \text { SumProdPerm }\left[A_{0}(5), A_{1}(0), A_{2}(2)\right]+\operatorname{SumProdPerm}\left[A_{0}(3), A_{1}(3), A_{2}(1)\right] \\
& + \text { SumProdPerm }\left[A_{0}(1), A_{1}(6), A_{2}(0)\right] ; h_{2}=3 h_{1} \\
& =\left\{\operatorname{SumProdPerm}\left[A_{0}(4), A_{1}(2), A_{2}(1)\right]+\operatorname{SumProdPerm}\left[A_{0}(1), A_{1}(6), A_{2}(0)\right] ; h_{2}=4 h_{1}\right. \\
& \operatorname{SumProdPerm}\left[A_{0}(5), A_{1}(1), A_{2}(1)\right]+\operatorname{SumProdPerm}\left[A_{0}(1), A_{1}(6), A_{2}(0)\right] ; h_{2}=5 h_{1} \\
& \operatorname{SumProdPerm}\left[A_{0}(6), A_{1}(0), A_{2}(1)\right]+\operatorname{SumProdPerm}\left[A_{0}(1), A_{1}(6), A_{2}(0)\right] ; h_{2}=6 h_{1} \\
& \text { SumProdPerm }\left[A_{0}(1), A_{1}(6), A_{2}(0)\right] \text {; otherwise } \\
& \text { Emerging pattern for } j=6, k \geq 6
\end{aligned}
$$$$
Q_{k}\left(6 h_{1}\right)=\left\{\begin{array}{l}
\sum_{r=0}^{3} \operatorname{SumProdPerm}\left[A_{0}(r+k-6), A_{1}(6-2 r), A_{2}(r)\right] ; h_{2}=2 h_{1} \\
\sum_{r=0}^{2} \operatorname{SumProdPerm}\left[A_{0}(2 r+k-6), A_{1}(6-3 r), A_{2}(r)\right] ; h_{2}=3 h_{1} \\
\sum_{r=0}^{1} \operatorname{SumProdPerm}\left[A_{0}(3 r+k-6), A_{1}(6-4 r), A_{2}(r)\right] ; h_{2}=4 h_{1} \\
\sum_{r=0}^{1} \operatorname{SumProdPerm}\left[A_{0}(4 r+k-6), A_{1}(6-5 r), A_{2}(r)\right] ; h_{2}=5 h_{1} \\
\sum_{r=0}^{1} \operatorname{SumProdPerm}\left[A_{0}(5 r+k-6), A_{1}(6-6 r), A_{2}(r)\right] ; h_{2}=6 h_{1} \\
\text { SumProdPerm }\left[A_{0}(k-6), A_{1}(6), A_{2}(0)\right] ; \text { otherwise }
\end{array}\right.
$$

3.1.6 Determining Matrices for $j=7, k \geq 2$

For $j=7, k=2$

$$
Q_{2}\left(7 h_{1}\right)=A_{0} Q_{1}\left(7 h_{1}\right)+A_{1} Q_{1}\left(6 h_{1}\right)+A_{2} Q_{1}\left(7 h_{1}-h_{2}\right)
$$




$$
\left\{\begin{array}{l}
0 ; h_{2}=2 h_{1} \\
0 ; h_{2}=3 h_{1} \\
0 ; h_{2}=4 h_{1} \\
0 ; h_{2}=5 h_{1} \\
\text { SumProdPerm }\left[A_{0}(0), A_{1}(1), A_{2}(1)\right] ; h_{2}=6 h_{1} \\
\text { SumProdPerm }\left[A_{0}(1), A_{1}(0), A_{2}(1)\right] ; h_{2}=7 h_{1} \\
0 ; \text { otherwise }
\end{array}\right.
$$$$
\text { For } j=7, k=3
$$$$
\begin{aligned}
& \quad Q_{3}\left(7 h_{1}\right)=A_{0} Q_{2}\left(7 h_{1}\right)+A_{1} Q_{2}\left(6 h_{1}\right)+A_{2} Q_{2}\left(7 h_{1}-h_{2}\right) \\
& 0 ; h_{2}=2 h_{1} \\
& \text { SumProdPerm }\left[A_{0}(0), A_{1}(1), A_{2}(2)\right] ; h_{2}=3 h_{1} \\
& 0 ; h_{2}=4 h_{1} \\
& \text { SumProdPerm }\left[A_{0}(0), A_{1}(2), A_{2}(1)\right] ; h_{2}=5 h_{1} \\
& \text { SumProdPerm }\left[A_{0}(1), A_{1}(1), A_{2}(1)\right] ; h_{2}=6 h_{1} \\
& \text { SumProdPerm }\left[A_{0}(2), A_{1}(0), A_{2}(1)\right] ; h_{2}=7 h_{1} \\
& 0 ; \text { otherwise }
\end{aligned}
$$$$
\text { For } j=7, k=4
$$$$
Q_{4}\left(7 h_{1}\right)=A_{0} Q_{3}\left(7 h_{1}\right)+A_{1} Q_{3}\left(6 h_{1}\right)+A_{2} Q_{3}\left(7 h_{1}-h_{2}\right)
$$ 


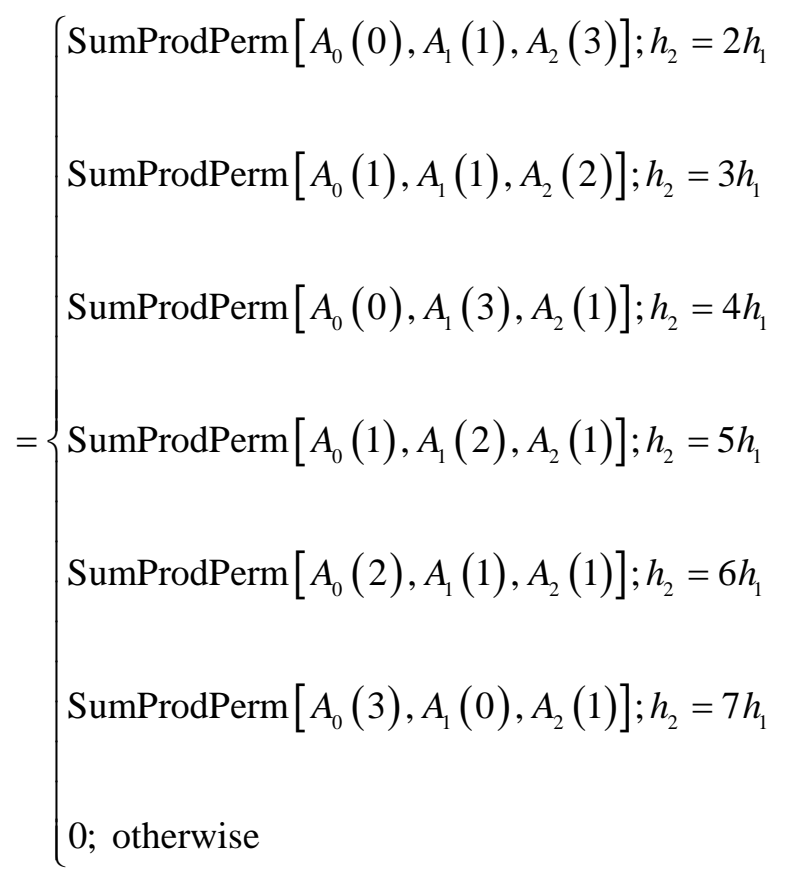

For $j=7, k=5$

$$
\begin{aligned}
& \qquad Q_{5}\left(7 h_{1}\right)=A_{0} Q_{4}\left(7 h_{1}\right)+A_{1} Q_{4}\left(6 h_{1}\right)+A_{2} Q_{4}\left(7 h_{1}-h_{2}\right) \\
& \text { SumProdPerm }\left[A_{0}(1), A_{1}(1), A_{2}(3)\right]+\operatorname{SumProdPerm}\left[A_{0}(0), A_{1}(3), A_{2}(2)\right] ; h_{2}=2 h_{1} \\
& =\left\{\begin{array}{l}
\text { SumProdPerm }\left[A_{0}(1), A_{1}(3), A_{2}(1)\right] ; h_{2}=4 h_{1} \\
\text { SumProdPerm }\left[A_{0}(2), A_{1}(2), A_{2}(1)\right] ; h_{2}=5 h_{1} \\
\text { Sum ProdPerm }\left[A_{0}(3), A_{1}(1), A_{2}(1)\right] ; h_{2}=6 h_{1} \\
\text { SumProdPerm }\left[A_{0}(4), A_{1}(0), A_{2}(1)\right] ; h_{2}=7 h_{1} \\
0 ; \text { otherwise }
\end{array}\right. \\
& \text { For } j=7, k=7
\end{aligned}
$$$$
Q_{7}\left(7 h_{1}\right)=A_{0} Q_{6}\left(7 h_{1}\right)+A_{1} Q_{6}\left(6 h_{1}\right)+A_{2} Q_{6}\left(7 h_{1}-h_{2}\right)
$$ 


$$
=\left\{\begin{array}{l}
\text { SumProdPerm }\left[A_{0}(3), A_{1}(1), A_{2}(3)\right]+\operatorname{SumProdPerm}\left[A_{0}(2), A_{1}(3), A_{2}(2)\right] \\
+ \text { SumProdPerm }\left[A_{0}(1), A_{1}(5), A_{2}(1)\right]+\operatorname{SumProdPerm}\left[A_{0}(0), A_{1}(7), A_{2}(0)\right] ; h_{2}=2 h_{1} \\
\text { SumProdPerm }\left[A_{0}(4), A_{1}(1), A_{2}(2)\right]+\operatorname{SumProdPerm}\left[A_{0}(2), A_{1}(4), A_{2}(1)\right] \\
+ \text { SumProdPerm }\left[A_{0}(0), A_{1}(7), A_{2}(0)\right] ; h_{2}=3 h_{1} \\
\text { SumProdPerm }\left[A_{0}(3), A_{1}(3), A_{2}(1)\right]+\operatorname{SumProdPerm}\left[A_{0}(0), A_{1}(7), A_{2}(0)\right] ; h_{2}=4 h_{1} \\
\text { SumProdPerm }\left[A_{0}(4), A_{1}(2), A_{2}(1)\right]+\operatorname{SumProdPerm}\left[A_{0}(0), A_{1}(7), A_{2}(0)\right] ; h_{2}=5 h_{1} \\
\text { SumProdPerm }\left[A_{0}(5), A_{1}(1), A_{2}(1)\right]+\text { SumProdPerm }\left[A_{0}(0), A_{1}(7), A_{2}(0)\right] ; h_{2}=6 h_{1} \\
\text { SumProdPerm }\left[A_{0}(6), A_{1}(0), A_{2}(1)\right]+\text { SumProdPerm }\left[A_{0}(0), A_{1}(7), A_{2}(0)\right] ; h_{2}=7 h_{1} \\
\text { SumProdPerm }\left[A_{0}(0), A_{1}(7), A_{2}(0)\right] ; \text { otherwise }
\end{array}\right.
$$$$
\text { For } j=7, k=8
$$$$
Q_{8}\left(7 h_{1}\right)=A_{0} Q_{7}\left(7 h_{1}\right)+A_{1} Q_{7}\left(6 h_{1}\right)+A_{2} Q_{7}\left(7 h_{1}-h_{2}\right)
$$$$
\left\{\begin{array}{l}
\operatorname{SumProdPerm}\left[A_{0}(4), A_{1}(1), A_{2}(3)\right]+\operatorname{SumProdPerm}\left[A_{0}(3), A_{1}(3), A_{2}(2)\right] \\
+\operatorname{SumProdPerm}\left[A_{0}(2), A_{1}(5), A_{2}(1)\right]+\operatorname{SumProdPerm}\left[A_{0}(1), A_{1}(7), A_{2}(0)\right] ; h_{2}=2 h_{1}
\end{array}\right.
$$$$
\text { SumProdPerm }\left[A_{0}(5), A_{1}(1), A_{2}(2)\right]+\operatorname{SumProdPerm}\left[A_{0}(3), A_{1}(4), A_{2}(1)\right]
$$$$
+ \text { SumProdPerm }\left[A_{0}(1), A_{1}(7), A_{2}(0)\right] ; h_{2}=3 h_{1}
$$$$
\text { SumProdPerm }\left[A_{0}(4), A_{1}(3), A_{2}(1)\right]+\operatorname{SumProdPerm}\left[A_{0}(1), A_{1}(7), A_{2}(0)\right] ; h_{2}=4 h_{1}
$$$$
=\{
$$$$
\text { SumProdPerm }\left[A_{0}(5), A_{1}(2), A_{2}(1)\right]+\operatorname{SumProdPerm}\left[A_{0}(1), A_{1}(7), A_{2}(0)\right] ; h_{2}=5 h_{1}
$$$$
\text { SumProdPerm }\left[A_{0}(6), A_{1}(1), A_{2}(1)\right]+\operatorname{SumProdPerm}\left[A_{0}(1), A_{1}(7), A_{2}(0)\right] ; h_{2}=6 h_{1}
$$$$
\text { SumProdPerm }\left[A_{0}(7), A_{1}(0), A_{2}(1)\right]+\operatorname{SumProdPerm}\left[A_{0}(1), A_{1}(7), A_{2}(0)\right] ; h_{2}=7 h_{1}
$$$$
\text { SumProdPerm }\left[A_{0}(1), A_{1}(7), A_{2}(0)\right] \text {; otherwise }
$$$$
\text { Emerging pattern for } j=7, k \geq 7
$$ 


$$
Q_{k}\left(7 h_{1}\right)=\left\{\begin{array}{l}
\sum_{r=0}^{3} \operatorname{SumProdPerm}\left[A_{0}(r+k-7), A_{1}(7-2 r), A_{2}(r)\right] ; h_{2}=2 h_{1} \\
\sum_{r=0}^{2} \operatorname{SumProdPerm}\left[A_{0}(2 r+k-7), A_{1}(7-3 r), A_{2}(r)\right] ; h_{2}=3 h_{1} \\
\sum_{r=0}^{1} \operatorname{SumProdPerm}\left[A_{0}(3 r+k-7), A_{1}(7-4 r), A_{2}(r)\right] ; h_{2}=4 h_{1} \\
\sum_{r=0}^{1} \operatorname{SumProdPerm}\left[A_{0}(5 r+k-7), A_{1}(7-6 r), A_{2}(r)\right] ; h_{2}=6 h_{1} \\
\sum_{r=0}^{1} \operatorname{SumProdPerm}\left[A_{0}(6 r+k-7), A_{1}(7-7 r), A_{2}(r)\right] ; h_{2}=7 h_{1} \\
\text { SumProdPerm }\left[A_{0}(k-7), A_{1}(7), A_{2}(0)\right] ; \text { otherwise }
\end{array}\right.
$$

From these expressions of the determining matrices for particular cases, a general expression for the determining matrices $Q_{k}\left(j h_{1}\right) ; 0 \leq j \leq k, k \neq 0$ and $Q_{k}\left(j h_{1}\right) ; j>k \geq 1$ can be formulated and proved.

\section{Conclusion}

The results on determining matrices derived here greatly extended the published works of Ukwu (2016) and Ukwu (2014). Moreover, the results here went further to examine the structure and computing complexity of the generic case of the double time-delay control systems that previous authors did not examine in this field in simpler systems. This work has successfully prosecuted a number of tasks; by exploiting relevant facts about the permutation of matrices already established in Ukwu (2014a) and deft application of mathematical induction principles, it is established that determining matrices for double timedelay linear autonomous functional differential control systems do not exist if one of the time-delays is an integer multiple of the other and the structures of the determining matrices for particular cases of the system in consideration follow certain patterns which can be further investigated to determine the Euclidean controllability of the system.

\section{References}

[1] Angell T. S. (1980). On Controllability for Nonlinear Hereditary Systems: A Fixed Approach, Nonlinear Analysis, Theory, Methods and Applications, 4, 529-548.

[2] Balachandran K. (1986). Controllability of Nonlinear Systems with Delays in both State and Control, Kybernetika, 22, 1-5.

[3] Balachandran K. (1992). Controllability of Neutral Volterra Integrodifferential Systems, Journal of Australian Mathematical Society Series, B34, 18-25.

[4] Balachandran K. and Balasubramaniam P. (1993). A Note on Controllability of Neutral Volterra Integrodifferential Systems, Journal of Applied Mathematics and Stochastic Analysis, 6, 153-160.

[5] Banks H. T. and Kent G. A. (1972). Control of Functional Differential Equations of Retarded and Neutral Type to targets in Function Space, SIAM Journal of Control Theory, 10, 1-5.

[6] Chang Y. K. and Chalishajar D. N. (2008). Controllability of Mixed Volterra-Fredholm type Integro Differential Inclusions in Banach Spaces, Journal of Franklin Institute, 345, 499-507.

[7] Chukwu E. N. (1992). Stability and Time-Optimal Control of Hereditary Systems, New York: Academic Press.

[8] Chukwu E. N. (2001). Differential Models and Neutral Systems for Controlling the Wealth of Nations. Series on Advances in Mathematics for Applied Sciences, New York Academic Press, 54. 
[9] Dauer J. P. and Gahl R. D. (1977). Controllability of Nonlinear Delay Systems, Journal of Optimization Theory and Applications, 21, (1), 1-3.

[10] Gabasov R. and Kirillova F. (1976). The Qualitative Theory of Optimal Processes, New York: Marcel Dekker Inc.

[11] Manitius A. (1978). Control Theory and Topics in Functional Analysis, International Atomic Energy Agency, 3.

[12] Onwuatu J. U. (1984). On the Null-Controllability in Function Space of Nonlinear Systems of Neutral Functional Differential Equations with Limited Controls, Journal of Optimization Theory and Applications, 42, 397-420.

[13] Selvi S. and Mallika A. M. (2012). Controllability Results for Impulsive Differential Systems with Finite Delay, Journal of Nonlinear Scientific Applications, 5, 206-219.

[14] Ukwu C. (1992). Euclidean Controllability and Cores of Euclidean Targets for Differential Difference Systems, North Carolina State University, Raleigh, USA: Unpublished Master of Science Thesis in Applied Mathematics with Operations Research.

[15] Ukwu C. (2014). The Structure of Determining Matrices for a Class of Double-Delay Control Systems, International Journal of Mathematics and Statistics Inventions (IJMSI), 2, (2), 14-30.

[16] Ukwu C. (2014a), The Structure of Determining Matrices for Single-Delay Autonomous Linear Neutral Control Systems, International Journal of Mathematics and Statistics Inventions (IJMSI), 2, (3), 31-47.

[17] Ukwu C. (2016). Functional Differential Systems: Structures, Cores and Controllability, Deutschland: LAP LAMBERT Academic Publishing.

[18] Ukwu C. (2016a). Necessary and Sufficient Conditions for Controllability of Double-Delay Autonomous Linear Control Systems, Journal of Scientific Research and Reports, 10, (3), 1-9.

[19] Ukwu C. and Garba E. (2014). Derivation of an Optimal Expression for Solution Matrices of a class of Single-Delay Scalar Differential Equations, Journal of the Nigerian Association of Mathematical Physics, 28-35.

[20] Underwood R. G. and Chukwu E. N. (1988). Null Controllability of Nonlinear Neutral Differential Equations, Journal of Mathematical Analysis and Applications, 129, 472-483. 